\title{
Metaloproteinasas y Osteoartritis
}

\author{
Marco Galleguillos C. BQ Mg BQ ${ }^{1}$, Raúl Carrillo G. Lic. MV ${ }^{1}$, Gladys Flores Lic. MV ${ }^{1}$, \\ Héctor Adarmes A. MV Mg BQ ${ }^{1}$ \\ ${ }^{1}$ Laboratorio de Bioquímica, Departamento de Ciencias Biológicas Animales, Facultad de Ciencias \\ Veterinarias y Pecuarias, Universidad de Chile. \\ Email:mgallegu@uchile.cl
}

\begin{abstract}
Resumen
Las metaloproteinasas (MMPs) son una familia de enzimas proteolíticas capaces de procesar o degradar proteínas de la matriz extracelular. Las MMPs tienen un prodominio, prodominio catalítico, una región bisagra y un dominio tipo hemopexina (excepto en las matrilisinas). Pueden ser activadas in vivo por otras MMPs, proteinasas tisulares o proteinasas plasmáticas. La regulación de estas enzimas puede ocurrir a nivel de su síntesis, secreción, activación del zimógeno. Los inhibidores tisulares de metaloproteinasas (TIMPs) son inhibidores específicos de las MMPs que participan en el control local de su actividad. En el cartílago normal existe bajo recambio de las moléculas de la matriz extracelular. Existe un delicado balance entre la síntesis y degradación de los componentes del cartílago articular. Este balance se pierde en enfermedades degenerativas como la osteoartritis en dónde participan algunas MMPs tales como las gelatinasas, estromelisinas y colagenasas. Estos procesos podrían ser blancos farmacológicos en el tratamiento de esta enfermedad.
\end{abstract}

Palabras clave: metaloproteinasa, osteoartritis, cartílago.

\section{Introducción}

La capacidad para degradar proteínas extracelulares es esencial para la interacción entre la célula con su ambiente, particularmente para los organismos pluricelulares. El proceso de recambio de la matriz extracelular (MEC) está involucrado en la morfogénesis, embriogénesis, reproducción, reabsorción y remodelación de tejidos. En el recambio tisular normal y alterado, que incluye en este último caso a procesos inflamatorios y tumorales, participan enzimas del tipo de las metaloproteinasas de matriz (MMPs) que pertenecen una familia de endoproteinasas secretadas en forma de zimógeno, cuya activación se produce por proteolisis parcial, con pérdida de un péptido de aproximadamente $10 \mathrm{kDa}$ de masa molecular. También se activan por cambios conformacionales provocados por otras proteinasas o por compuestos organomercuriales y son inactivadas por una familia de inhibidores tisulares específicos (TIMPs), que se unen al sitio activo a través de interacciones no covalentes. Estas enzimas poseen una amplia distribución, tanto en organismos vertebrados como en invertebrados y probablemente aparecieron tempranamente en la evolución. Entre las características generales se encuentran: la presencia de $\mathrm{Zn}+2$ en su estructura, la capacidad de degradar uno o más componentes de la MEC, la presencia de los residuos de ácido aspártico e histidina esenciales para su actividad hidrolítica, el requerimiento de $\mathrm{Ca}+2$ para su actividad y el $\mathrm{pH}$ óptimo de catálisis entre 7 y 8 
(Sternlicht y Werb, 2001). Esta revisión general trata de las MMPs y su relación con la osteoartritis.

\section{Clasificación de las MMPs}

Se han identificado 26 miembros de la familia de las MMPs en vertebrados que se dividen en seis grupos de acuerdo a su especificidad de sustrato:

1. Las colagenasas que hidrolizan preferentemente fibras de colágeno de tipo I, II, III, VII, VIII y X.

2. Las gelatinasas que degradan el colágeno tipo IV y $\mathrm{V}$ y la proteína central del agrecán, que es el proteoglicano más común y de mayor tamaño de la MEC del cartílago articular.

3. Las estromelisinas que degradan una variedad de moléculas de la matriz como proteoglicanos, laminina, fibronectina y además activan los zimógenos de la MMP1, MMP3, MMP8, MMP9 y MMP13.

4. Las matrilisinas que incluyen a la matrilisina-1 (MMP7) y matrilisina-2 (MMP26, endometasa). La MMP7 procesa moléculas de la superficie celular tales como pro- -defensina, Fas-ligando, Pro-TNF- y E-caderina.

5. Las MMPs asociadas a membranas ( MT-MMPs) incluyen a un grupo menos numeroso pero con cierta relevancia en la activación de los zimógenos de algunas MMPs. Así, las MT-MMP14 y MT-MMP15 activan las formas latentes de la MMP2 y MMP13 (Woessner, 1991; Parsons y col., 1997; Nagase y Woessner; 1999, Sternlicht y Werb, 2001; Fanjul-Fernández y col., 2010).

6. Otras MMPs descritas no clasificadas como la MMP12 expresada en macrófagos en dónde participa en la migración celular. Otras enzimas dentro de este grupo son las MMP19, MMP20, MMP21, MMP22, MMP23, MMP27 y MMP28.
Aunque cada MMP tiene sustratos específicos, existe una superposición de otros sustratos de tal modo que en conjunto son capaces de abarcar una gran variedad de componentes de la MEC (Clutterbuck y col. 2010).

Desde el punto de vista estructural las MMPs tienen un prodominio, un prodominio catalítico, una región bisagra y un dominio tipo hemopexina. Este último dominio no se encuentra en las matrilisinas. Las MMPs poseen además tres características moleculares (Raffetto y Khall, 2008; Sternlicht y Werb, 2001; Visse y Nagase 2003):

1. Una secuencia de homología a colagenasa-1 (MMP1).

2. Un motivo PRCGXPD "switch" de cisteína en el prodominio que mantiene a la MMP en su forma de zimógeno (proMMP). Esta cisteína conservada quela el $\mathrm{Zn}+2$ del sitio catalítico.

3. Un motivo de unión a $\mathrm{Zn}+2$ unido a tres histidinas con una secuencia conservada HEXGHXXGXXH localizada en el dominio catalítico.

\section{Activación de las proMMPs}

La activación in vivo de la mayoría de las proMMPs se produce posiblemente por proteinasas tisulares, plasmáticas o bien por proteinasas bacterianas oportunistas (Ogata y col., 1995; Nagase y Woessner, 1999). El óxido nítrico (NO) puede activar la proMMP9 durante la isquemia cerebral por reacción con el grupo tiol del "switch" de cisteína y formar un derivado S-nitrosilado (Gu y col., 2002).

La MMP3 activa varias de proMMPs entre las cuales se encuentra la proMMP1. La proMMP2 no es activada en la superficie celular por proteinasas generales, sino más bien por MT-MMPs, exceptuando la MT4-MMP. La MT1-MMP activa a la proMMP2 con la participación del TIMP-2 (Wang y col., 2000). La proMMP2 forma un complejo con TIMP2 a través de su dominio C-terminal permitiendo así que el dominio inhibitorio N-terminal de TIMP2 se una a MT1-MMP sobre la superficie celular. La proMMP2 
unida a la superficie celular es activada por la MT1MMP libre de TIMP2. Alternativamente, la MT1MMP inhibida por TIMP2 puede actuar como un receptor de la proMMP2. El complejo MT1-MMPTIMP2-proMMP2 se presenta a una MT1-MMP libre para su activación. El agrupamiento de MT1-MMP sobre la superficie celular a través del dominio hemopexina facilita este proceso de activación.

\section{Regulación de la Actividad de las MMP}

La actividad de las MMPs requiere de un alto grado de regulación a distintos niveles debido a su participación en diversos procesos fisiológicos. Pueden ser reguladas a nivel transcripcional y postranscripcional (Sternlicht y Werb, 2001; Clutterbuck y col., 2010). La expresión de la mayoría de las MMPs es regulada transcripcionalmente por factores de crecimiento, hormonas, citoquinas, incluidas las interleuquinas, interferón, TNF- y TNF- (Sternlicht y Werb, 2001). El nivel postranscripcional corresponde a la activación de sus precursores (proMMPs) así como la inhibición, tanto del proceso de activación mismo como de sus formas activas por la acción de inhibidores endógenos que incluyen a la 2macroglobulina e inhibidores tisulares de metaloproteinasas (TIMPs) (Ogata y col., 1995). Hasta la fecha en vertebrados se han identificado cuatro TIMPs (TIMP-1, TIMP-2, TIMP-3 y TIMP-4) los cuales se unen a las MMPs con una estequiometria 1:1 y su expresión es regulada durante el desarrollo y la remodelación de tejido (Visse y Nagase, 2002). Una producción excesiva o inapropiada de MMPs puede contribuir en la patogénesis de varios procesos degradativos de los tejidos, que incluyen a la osteoartritis (OA) y otras enfermedades altamente prevalentes tales como: esclerosis múltiple, pérdida de dientes, progresión y metástasis tumoral, enfermedad obstructiva crónica (Shapiro, 1998; Tallant y col., 2010). También se incluyen enfermedades cardiovasculares, como la arterioesclerosis y la formación de aneurismas (Raffetto y Khalil, 2008; Siefert y Sarkar, 2012; Tallant y col., 2010).

\section{Metaloproteinasas y osteoartritis}

La osteoartritis (OA) es una enfermedad articular crónica degenerativa multifactorial que afecta a millones de personas en el mundo. Se caracteriza por cambios en la articulación completa que conducen a la degradación del cartílago articular, inflamación de la membrana sinovial (sinovitis), en el hueso subcondral y crecimiento de nuevo hueso y cartílago (osteofitos) en el borde de la articulación. En un cartílago sano existe un balance entre la actividad catabólica y anabólica con un bajo recambio de los componentes de la MEC. En la OA el condrocito es estimulado a producir enzimas proteolíticas, lo cual lleva a una destrucción focal del cartílago y en algunos casos a la inflamación de la membrana sinovial (Moeller y Tuan, 2011).

Se ha descrito la presencia de MMPs en la membrana sinovial y en el cartílago articular de equino, principalmente de estromelisina (MMP-3), que a su vez posee una gran homología estructural con la MMP-3 de otras especies (Richardson y Dodge, 1998; Balkman y Nixon, 1998). La lesión de la membrana sinovial provoca la liberación hacia el medio extracelular y en particular hacia el líquido sinovial, de nucleótidos de adenina (ATP, ADP), enzimas lisosomales, prostaglandina E2, radicales libres, citoquinas, entre las que se puede encontrar principalmente interleuquina 1 (IL-1 ) y el factor de necrosis tumoral alfa (TNF). Todas estas moléculas a su vez estimularían la liberación de MMPs como colagenasa, estromelisina y gelatinasa desde condrocitos, sinoviocitos, fibroblastos y macrófagos infiltrados debido al proceso inflamatorio. Estas MMPs degradarían los proteoglicanos y el colágeno tipo II, que constituyen los componentes orgánicos cuantitativamente más importantes del cartílago (Cornelissen y col., 1998).

A pesar de que los tejidos normales sintetizan MMPs para los procesos de recambio normal, un desbalance entre su actividad y la concentración de TIMPs puede aumentar el daño articular (Morris y Treadwell, 1994; Cornelissen y col., 1998; Clutterbuck y col, 2010). Se ha descrito que en el líquido sinovial la pérdida del equilibrio entre las concentraciones de MMP3 (estromelisina-1) y de TIMP-1 determina la conversión 
de la molécula de progelatinasa en la forma activa (Watanabe y col., 1999). El desbalance entre la síntesis y la degradación puede generar una pérdida neta de cartílago articular, evidenciándose un aumento significativo en la actividad de MMPs en el líquido sinovial de animales con una alteración articular en comparación con animales normales (Brama y col., 1998; Cawston, 1998).

Las MMP-2 y MMP-9, conocidas también por gelatinasas, se encuentran elevadas en líquido sinovial proveniente de varias enfermedades articulares en humano (Hirose y col., 1992; Ahrens y col. 1996), perro (Coughlan y col., 1998) y en equino (Clegg y col., 1997b; Clegg y Carter, 1999). La producción de MMP2 y MMP9 en equino se ha demostrado en tejido articular y células inflamatorias infiltradas (Clegg y col., 1997a). Estas enzimas degradan el colágeno desnaturalizado (Hirose y col, 1992), proteína central del agrecán (Fosang y col., 1992), proteína de unión al cartílago (Nguyen y col., 1993) y el agrecán (Lark y col. 1995). Se ha encontrado la forma dimérica de MMP9 en líquido sinovial proveniente de articulación con artritis séptica y en una proporción directa con el recuento celular de la serie blanca (Clegg y col. 1997b).

La colagenasa 3 o MMP13 tiene un papel clave en la degradación del cartílago puesto que muchas vías catabólicas aumentan su actividad. Esta enzima es muy activa en la degradación del colágeno tipo II y se observado en un modelo quirúrgico de $\mathrm{OA}$ que ratas "knockout" para ese gen son más resistentes a la erosión del cartílago en comparación a sus animales control (Little y col., 2009). Se ha observado en ratas transgénicas que una sobre expresión de la MMP13 provoca una degeneración del cartílago comparable a la ocurrida en la OA (Neuhold y col., 2001). Esta enzima además de degradar el colágeno II, es capaz de degradar el agrecán (Fosang y col., 1996). El TIMP-3 tiene una acción reguladora importante in vivo como lo refleja el experimento con ratas "knockout" para este gen quienes presentaron una degradación del cartílago similar a la OA además de encontrarse una mayor concentración sérica de productos de degradación del colágeno II (Sahebjam y col., 2007).

\section{Concusiones}

Las MMPs tienen un papel importante en la degradación del cartílago articular ocurrida en la OA lo cual implica que son un potencial blanco de nuevos fármacos como tratamiento de este proceso patológico. Sin embargo, debido a la semejanza estructural de las MMPs, particularmente en su sitio activo, es necesario lograr una mayor especificidad en la acción de estos posibles fármacos. Es importante destacar que se requiere controlar la inflamación reiterada en la articulación para evitar la generación de este círculo vicioso en perpetúa la expresión y activación de MMPs.

\section{Referencia}

1. Ahrens, D.; Koch, A.E.; Pope, R.M.; SteinPicarella, M.; Niedbala, M.J. (1996) Expression of matrix metalloproteinase 9 (96-kd gelatinase B) in human rheumatoid arthritis. Arthritis Rheum. 39:1576-1587.

2. Balkman, Ch.E.; Nixon, A.J. 1998. Molecular cloning and cartilage gene expression of equine stromelysin 1 (matrix metalloproteinase 3). Am J Vet. Res. 59 (1): 30-36.

3. Brama Paj, Tekoppele Jm, Beekman B, Van Weeren Pr, Barnebeld A. 1998. Matrix metalloproteinase activity in equine synovial fluid: Influence of age, osteoarthritis, and osteochondrosis. Ann Rheum Dis. 57: 697-699.

4. Cawston Te. 1998. Matrix metalloproteinases and TIMPs: properties and implications for the rheumatic diseases. Mol Med Today 4(3): 130137.

5. Clegg Pd, Burke Rm, Coughlan Ar, Riggs Cm, Carter Sd. 1997a. Characterization of equine matrix metalloproteinase 2 and 9; and identification of the cellular sources of these enzymes in joints. Equine Vet J. 29 (5): 335-342.

6. Clegg Pd, Coughlan Ar, Riggs Cm, Carter Sd. 1997b. Matrix metalloproteinases 2 and 9 in equine synovial fluids. Equine Vet. J. 29(5): 343348. 
7. Clegg Pd, Carter Sd. (1999) Matrix metalloproteinase-2 and -9 are activated in joint diseases. Equine Vet. J. 31(4):324-30.

8. Clutterbuck Al, Harris P, Allaway D, Mobascheri A. 2010, Matrix metalloproteinases in inflammatory pathologies of the horse. Vet. J. 183(1): $27-38$

9. Cornelissen Bp, Rijkenhuizen Ab, Van Den Hoogen Bm, Rutten Vp, Barneveld A. 1998. Experimental model of synovitis/capsulitis in the equine metacarpophalangeal joint. Am. J. Vet. Res. 59(8):978-985.

10. Coughlan Ar, Robertson Dh, Bennett D, May C, Beynon Rj, Carter Sd. 1998. Matrix metalloproteinases 2 and 9 in canine rheumatoid arthritis. Vet Rec. 143(8): 219-223.

11. Fanjul-Fernández M, Folgueras A, Cabrera S, López-Ótin C. (2010) Matrix metalloproteinases: evolution, gene regulation and functional analysis in mouse models. Biochimica et Biophysica Acta. 1803(2010): 3-19

12. Fosang Aj, Last K, Knauper V, Murphy G, Neame Pj. 1996. Degradation of cartilage aggrecan by collagenase-3 (MMP-13). FEBS Lett. 380:17-20.

13. Fosang $\mathrm{Aj}$, Neame $\mathrm{Pj}$, Last $\mathrm{K}$, Hardingham $\mathrm{Te}$, Murphy G, Hamilton J. 1992. The interglobular domain of cartilage aggrecan is cleaved by PUMP, gelatinases, and cathepsin-B. J Biol Chem 267: 19470-19474.

14. Gu Z, Kaul M, Yan B, Kridel Sj, Cui J., Strongin A., Smith Jw, Liddington Rc, Lipton Sa. 2002. Snitrosylation of matrix metalloproteinases: signaling pathway to neuronal cell death. Science. 297:1186-1190.

15. Hirose T, Reife Ra, Smith Gn Jr, Stevens Rm, Mainardi Cl, Hasty Ka. 1992. Characterization of type $\mathrm{V}$ collagenase (gelatinase) in synovial fluid of patients with inflammatory arthritis. J Rheumatol. 19:593-599.

16. Lark Mw, Bayne Ek, Lohmander Ls. 1995. Aggrecan degradation in osteoarthritis and rheumatoid arthritis. Acta Orthop Scand Suppl. 266:92-97.

17. Little $\mathrm{Cb}$, Barai A, Burkhardt D, Smith Sm, Fosang Aj, Werb Z, Shah M, Tyhompson Ew. 2009. Metalloproteinase 13-deficient mice are resistant to osteoarthritis cartilage erosion but not chondrocyte hypertrophy or osteophyte development. Arthritis Rheum. 60:3723-3733.

18. Moeller Mb, Tuan R. 2011. Anabolic/catabolic balance in pathogenesis of osteoarthritis: identifying molecular targets. MR R. 3(6 Suppl 1): S3-S11.

19. Morris Ea, Treadwell Bv. 1994. Effect of interleukin 1 on articular cartilage from young and aged horses and comparison with metabolism of osteoarthritic cartilage. Am J Vet Res. 55(1): 138146.

20. Nagase H, Woessner Jf Jr. 1999. Matrix Metolloproteinases. J Biol Chem. 274(31): 2149121494.

21. Neuhold La, Killar L, Zhao W, Sung Ml, Warner L, Kulik J, Turner J, Wu W, Billinghurst C, Meijers T, Poole Ar, Babij P, Degennaro Lj. 2001. Postnatal expression in hyaline cartilage of constitutively active human collagenase-3 (MMP13) induces osteoarthritis in mice. J Clin Invest. 107:35-44.

22. Nguyen Q, Murphy G, Hughes Ce, Mort Js, Roughley Pj. 1993. Matrix metalloproteinases cleaves at two distinct sites on human cartilage link protein. Biochem J. 295:595-598.

23. Ogata Y, Itoh Y, Nagase H. 1995. Steps Involved in Activation of the Pro-matrix Metalloproteinase 9 (Progelatinase B)-Tissue Inhibitor of Metalloproteinases-1 Complex by 4Aminophenylmercuric Acetate and Proteinases. J Biol Chem. 270(31):18506-18511.

24. Parsons Sl, Watson Sa, Brown Pd, Collins Hm, Steele Rjc. 1997. Matrix metalloproteinases. Br. J. Surg. 84:160-166.

25. Raffetto Jd, Khalil Ra. 2008. Matrix metalloproteinases and their inhibitors in vascular remodeling and vascular disease. Biochem Farmacol 75:346-359. 
26. Richardson Dw, Dodge Gr. 1998. Molecular characteristics of equine stromelysin and the tissue inhibitor of metalloproteinase-1. Am J Vet Res. 59 (12):1557-1562.

27. Sahebjam S, Khokha R, Mort Js. 2007. Increased collagen and aggrecan degradation with age in the joints of Timp3(-/-) mice. Arthritis Rheum. 56:905-909.

28. Siefert Sa, Sarkar R. 2012. Matrix metalloproteinase in vascular physiology and disease. Vascular 20(4): 210-216.

29. Sternlicht, M.; Werb, Z. 2001.How matrix metalloproteinases regulate cell behavior. Annu. Rev. Cell Dev. Biol. 17:463-516.

30. Tallant C, Marrero A, Gomis-Ruth X. 2010. Matrix metalloproteinases. Fold and function of their catalytic domains. Biochimica et Biophysica Acta. 180: 20-28.

31. Visse R, Nagase H. 2003. Matrix metalloproteinase and tissue inhibitors of metalloproteinases: structure, function, and biochemistry. Circ Res. 92: 827-839.

32. Wang Z, Juttermann R, Soloway Pd. 2000. TIMP2 is required for efficient activation of proMMP-2 in vivo. J Biol Chem. 275:26411-26415.

33. Watanabe $\mathrm{Y}$, Yamaguchi R, Iwaki-Egawa S, Shimamori Y, Fujimoto Y, Matsuno H. 1999. Activation of progelatinase B in synovial fluids of patients with rheumatoid arthritis, with reference to stromelysin-1 and tissue inhibitor of matrix metalloproteinase-1. Clin Exp Rheumatol. 17(4):401-406.

34. Woessner, J.F. 1991. Matrix metalloproteinases and their inhibitors in connective tissue remodeling. FASEB J. 5: 2145-2154. 